\title{
Incremental Construction of a Lexical Transducer for Korean $^{1}$
}

\author{
Hyuk-Chul Kwon*, Lauri Karttunen \\ 1)ept. of Computer Science, Pusan National Univ. Pusan, 609-735, South Korea* \\ Xcrox PARC, 3333 Coyote Fill Road,Palo Alto C $\Lambda 94304$
}

\begin{abstract}
The paper describes the construction of a lexical transducer for Korcan that can be used for stemming and generation. 'The method contains two innovations: (1) two-level rules as well-formedness constraints in the initial phase; (2) the combination of intersection and composition of rule transducers in a decp cascade for the final result.
\end{abstract}

\section{Keywords}

Korean Iscxical 'Iransducer, 'Two-level Morphology, Morphotactics, Ordered liules

\section{Introduction}

This paper presents an incremental construction method of a lexical transducer (LC) for Korcan. A lexical transducer, first described by Kartiunen, Kaplan, and 7aenen (KK\%) (Karttunen, 1992a), is a specialized finite-state transducer (FST) that maps canonical citation forms of words and morphological categories to inflected surface forms, and vice versa. IT's have many advantages for stemming, morphological analysis, and generation. 'They are

(i) bidirectional: the sarne structure can be used for sternming and gencration.

(ii) efficient: the recognition and generation of word forms docs not require the application of any morphological rules at runtime.

L'I's for Jinglish and Fench have been built at Xorox $P A R C$ within a framcwork known as two-level morphology (Koskenniemi, 1983). As described by KKZ(Kartunon, 1992a), this can be done in three steps: (i) we construct a simple finite-state automaton ( $L A$ ) that defines all valid lexical forms (LF's) of the language. A LF is a concatenation of stcms and morphemes in their canonical dictionary representation. (ii) Wo describe morphological alternations by moans of two-level rules(Koskenniemi, 1983; Kart-

1 'lhis paper was partially supported by Korean Science and Lingineering Joundation. tunen, 1993), compile the rules to finite-state transducers, and intersect them to form a single rule transducer $\left(R^{r} \Gamma\right)$. (iii) We merge the $\mathrm{C} \Lambda$ with the $R^{t} \Gamma$ by composition producing the LI that has on its lexical side every valid lexical form of the language and on the surface side the corresponding realization as determined by the morphological alternations of the language.

KKZ argued that for lirench, it was best to divide step (ii) into two stagcs. $\Lambda$ threc-level description was required to give a linguistically satisfactory account of the plural formation of compound nouns. KKL opted for two cascading two-level rule systems that are cornpiled separately, then intersected laterally and finally composed to a single R' $\mathrm{L}$.

The task of building a morphological analyzer for a language such as Korean or Japanese is a much higher challenge than it is for English and l'rench. $A \mathrm{Ko}$ rean verb may have more than fifty thousand inflected forms. ${ }^{2}$ The Korean writing system (Ilangul) does not consistently distinguish botween single and compound nouns. Becanse Ilangul uses syllabic characters, changes in syllable structure are directly reflected in the orthography.

Because of the complexity of the morphological alternations in Korean, it is very difficult, although not impossible in principle, to describe them in a single two-level rule system or in a system that is limited to just three levels like the KKZ system for Fench. The most natural description of the Korcan alternation is a cascade of rules of greater depth.

\section{Morphological Alternations in Korean}

The Inangul is a phonemic syllable-based script where morphological alternations that change the syllable structure of the word are reflected in the orthography (Korean Ministry of Liducation, 1988; Kim, 1990). 'This paper uses the so-called Yale system for representing llangul in a Romanized form, except that wo

\footnotetext{
"A "e-jel(word)" which is a spacing unit of Mangul can consist of a verb stem, several endings and postpositions. 'The $\Lambda$.I Jab of I ept. of Compuler Science, Pusan National Univ. has more thare 50,000" $0-j c l "$ generated from "mek-ta(eat)"
} 
use wue and oa instead of we and wa of the Yale systom because we and wa do not show that they are diphthongs, composed of wne and $e$ and of $o$ and $a$ respectively.

lixamples (1) and (2) involve three simple morphological altemations: (i) the realization of a stem final $p$ in irregular predicates as a vowel in front of vowel-initial suflixes; (ii) left-to-right, vowel harmony based on partitioning of vowels into 'light' ([-+lighti]: $a$, $o$, oa), 'clark' and 'neutral'([-light] $)$; (iii) the realization of a morpheme boundary as a syllable boundary or as nothing.

A syllable boundary is introduced before the last consonant of irregular $-p$ verbs/ardjectives when a vowel-initial suflix follows and the -p itself is realized as $O$ if the preceding vowel is [+light], otherwise $w u$ by vowel harmony. Only some of the predicates ending in -p are irregular. In verbs that end in a vowel such as cwn 'to give', the vowel may mergo with a suffix-initial vowel to form a diphthong or it may retain its syllabic, status in a two-vowel sequence.

We use " $t$ " in the lexical representation to mark morphone boundaries, "_" to mark syllable boundaries, "()" to represent, deletion (surface side) and cpenthesis (lexical side), and two diacritic markers $\{p$ Verb $\}$ for an irregular $-p$ verb and $\{r$ Verb $\}$ for a regular verb to represent classes of verbal stems.

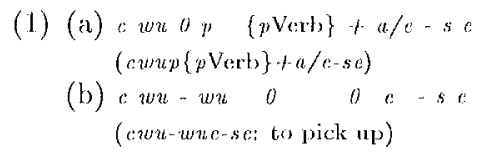

(2) (a) c wu $\{r \mathrm{Verb}\}+a / e-s e($ cwu $\{r \mathrm{Verl})\}-r a / e-s e)$

(b) $a$ wu 0 - e $-s:(c u u-e-s c$ : to give $)$

(c) c wh 0 o 0 - se (cuuc-se: to give)

The (a) part of both (1) and (2) are lexical forms and (b) and (c) are corresponding surface words.

Because cwup is an irregular - $p$ verb, the following phonemo $a / e$ is a vowel and the preceding syllable wu is [-light], $p$ in (1) (a) is realized as wu. The $a / o$ is realized as $\theta$ because the preceding surface vowel wh is [-light]. At the same time, wh and $e$ are contracted into a diphthong wue which is described as the deletion of "f" in (a) of (1). "These two changes are linked in that one inust not be allowed to hapeen without the other. Othorwise cwu-unt-e-se and curu-mue-se wonld be generated, but only cwu-wue-se is grammatical. On the other hand, in the case of the regular verb cwu, both cwu-e-se and the contracted variant cume-se are acceptablo.

These rules ean be described easily by two-level morphology as follows.
(3) (i) $\Lambda$ syllable boundary ("-") is introduced before a sterm-final $p$ in irregular $-p$ verbs/adjectives when a vowol-initial suffix follows.

(ii) A stem final $p$ in irrogular - $p$ verbs/adjectives is realized as o if the preceding vowel is [flight], otherwise $w u$.

(iii) $a / e$ is realized as $a$ if the precoding vowel is [t-light], otherwise $e$.

(iv) (a) The morphemo boundary following irregular - $p$ verbs/adjectives is deleted be fore a vowel-initial sulfix and realized as sylleble boundary elsewhere.

(b) 'The morpheme boundary in regulas: verbs/adjectives can be deleted or realized as a syllable bondary depending on context.

With the help of the Xerox two-level rule compiler ('twole')(Kartitunen, 1992b) the rules can be compiled to finite state transducers and intersected to a single transducer. Describing such phenomena as parallel rules may be complicated beciuse each fule may be a formutation of effectis caused by several phonological rules. Hor example, in formalizing (ii) as a two-level rule we must take into account both irregular conjugation of - $p$ verbs/adjectives and vowel harmony. 'This is a not a clesirable state of affairs. Wo will come back to this point later.

\section{Construction of a Korean Lexical Transducer(LT)}

'T'he first step in the construction of a lexical transducer is to create a sirmple finite state antomaton for all valid texical forms of Korean. 'The lexical antomaton $(L \Lambda)$ is composed with the first set of rule transducers (R'I'). 'J'he resulting transdueer has on its "upper" side, the valid lexical forms, and on the "lower" side, intermediate representalions derived by the lirst set of rules. 'This intermediate t,ansducer is composed with the second set of rule transducers and the procoss is iterated several times. At cach stage in the process, the lexical side remains unchanged and the intermediate representations are changed by the now set of rules. The final result is a transducer that associates the valid lexical forms with their proper surface realizations. Conceptually this is sirnilar to what happens in a traditional phonological derivation. Ilowever, note that rules apply to the lexicon as a whole. rather than to individual words and the result of each application is a new transducer. Because the intermediate levels disappear in the composition, the resulting IT' is equally well suited for morphological analysis as it is for generation.

The compilation and intersection of rule trauseluccrs was done with the fwole compiler, the construction 
of the $L \Lambda$ and the compositions we carried out with the Xerox interactive finite-state calculus ('ifsm').

\subsection{Construction of Lexical Automa- $\operatorname{ton}(\mathrm{L} A)$}

'The ifsm-utility enabled us to assemble the $L \Lambda$ incrementally. The first step was to divide the total list of morphemes into sublexicons on the basis of their morphological type and to make a tcxt file for each sublexicon. We added diacritic markers to the edges of certain types of morphemes in order to be able to cnforce morphotactic constraints on valid morpheme sequences.

Fach sublexicon was compiled separately to a finitestate automaton. The sublexicons were used to construct the LA with the help of the regular expression facility in the ifsm-toolkit. For example, having compiled a simple automaton from the list of simple nouns, we could expand it to an infinite lexicon of compound nouns with the regular expression

"noun.auto" [\# "noun.auto"]*

This regular expression reads the noun automaton from a file and concatenates it with itself any number of times and marks the internal word boundaries with \#.

'The first version of the LA was made in this way by combining sublexicons with regular operations (concatenation, union, iteration).

In order to enforce morphotactic constraints on the concatenation of some classes of suffixes, we wrote a set of two-level rules that require or prohibit the occurrence of particular diacritics at certain suffix boundaries. Lexical forms that do not satisfy the morpholactic constraints get eliminated in the composition with the well-formedness rules. The diacritics themselves are realized as zero so that they are not present in the lower side of the resulting transducer. The final form of the lexical automaton is obtained by extracting the lower-side from that transducer as a simple autornaton.

We believe that this incremental method of lexicon construction is better suited to morphologically complex languages than the lexicon format commonly used in two-level morphology. In standard two-level lexicons, individual entries contain information about which sublexicon they may concatenate with. The entire lexical structure is compiled in one step to large letter tree (Karttunen, 1993; Antworth, 1990). Our method is more tractable in two ways. Firstly, the lcxicon can be developed and refined stepwisc. Secondly, the morphotactic rules of the language are described explicitly as the regular expressions that construct the $\mathrm{L} \Lambda$ in conjunction with the well-formedness constraints that eliminate certain types of concatenations. In two-lcvel lexicons of the standard variety, the morphotactic structure of the language is not described explicitly at all. Rather, it is expressed in a very opaque and indirect way, in the sequences of links between entries and sublexicons.

Sproat argued that two-level morphology of morphotactics leads to a somewhat inelegant model of long-distance dependencies and suggested the unfication scheme, due to Bear, as a solution (Sproat, 1992). But unification scheme introduces additional runtime overhead. The above approach can easily and explicitly describe the fact that "-able" attaches to verbs formed with the prefix "cn-" and does not requirc additional runtime overhead.

We give a few examples of the difficulties in the description of Korean morphotactics. 'l'here are two different types of endings: (i) non-final (verbal) cndings for tense, modality, subject honorific or aspect, and (ii) final (verbal) endings as complementizer, nominalizer and adjectivizer. The non-final endings are placed in front of final cndings and must be followed by a suftix of the second type.

(4) shows the ordering restrictions of non-final endings. The parentheses indicate optionality.

(4) $(+$ IIon $)(+$ Past + Perf $(+$ Will $)$

$\{(+$ Past $)(+$ Will) (+ Retro $))$

(Hon:Honorific; Retro:Retrospection;

Perf: Perfect Aspect)

(4) compiles to a lexicon covering 20 different compound non-final ending sequences including null. This representation is clearly rrore informative than a simple listing of the members of the class. The prohibition of "Past+Perf+Will+Retro" in (4) can not be described by an adjacency table.

In (4) we do not need any morphotactic diacritics on the left because all non-final endings can combine with any verb and adjective stems and the combination of non-final and final endings is controlled by the diacritics of the latter group.

(5) shows three entries in the sublexicon of final endings. The elements in square brackets are morphotactic diacritics. (Square brackets indicate grouping, the vertical bar marks a disjunction.) The diacritics are deleted by well-formedness rules when the final endings are combined with other morpherres. 'I'he diacritics on the left of nun and nun-ka shows that they can not combine with adjectives.

(5) [Verb $|\Lambda \mathrm{dj}|$ Hon $\mid$ Past $\mid$ Will $\mid$ P'erf $]$ t a \{ Dec $\}$;

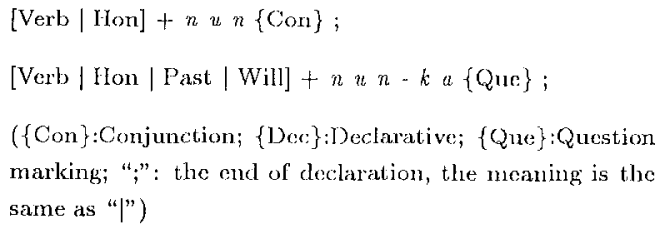


The diacritic markers $\{D$ ec $\},\{$ Que $\}$ and $\{C o n\}$ have two roles as the feature of the morphemes and as the right-hand context. 'They remain in linal $\mathrm{L} A$ because they are the feature of each morpheme.

By concatenating the sulonetworks of compound non-final endings and final endings, wo get a subtexicon of ending secuence as shown in (6). The [Vert) | Adj] diacrities indicate that non final endings can combine with any verls stems and adjective stems.

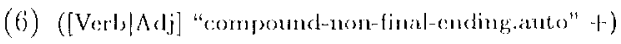
"Iinal ending. aut to"

'This concatenation produces an initial lexicon of $97198(2 * 20 * 2378+2378)$ different sequences where 20 is the number of compound non-final endings and 2378 is the number of secueneses of final endiugs with their morphotactic diacritics. 'This set is reduced to 7888 by a set of well-formedness rules that climinate unwanted sequences and delete the morphotactic dia critics. The composition of the intial lexicon with the well-formedness rules produces a transelucer from which the lower side is extracted as a simple antoma ton and used in the construction of the final $1 . \Lambda$.

Allowing nouns to freely compound with nouns creates a problen because it gives rise to many unacept. able or mnlikely compounds. fror example, the form coung-kan-i hats live alternate an alyses:

(7) cwung-kan-i

(a) camang-kan (niddle) +i(sul),ject, marker)

*(b) cusung-kan (middle) : : i (louse)

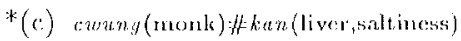
$+i$ (siubject marker)

*(d) cunng(nonk) \#l-ken(liver, saltiness) :Hi i looth, louse)

*(c) cumns(monk) \#kan-i(hamdiurss)

Our solution was to constrain compounding with a woll-formedness rule that excludes compounds with monosyllabic nouns (Kwon, 1990). 'llhe complexity of the morphological alternations in Korean is so high that, we need an easy way to give constraints incrementally. Our approach is a consistent and explicit way of describing mopholactic moles including long-distance dependencios.

\subsection{Composition of rexical Automa ton with Rule 'Iransducers}

After constructing the Korean I, $A$, we derive from it a lexicat dranselucer by composing the L $\mathrm{A}$, with rule t.ransiducers (RT's) in several stages. At each staige the previons result is composed with an R'l' derived by in. tersection from several two-level rules. Ihe rule sets include (i) morpheme generation rules, (ii) rules for irregular verbs/adjectives, (iii) deletion rules, (iv) vowel harmony rules and (v) contraction rules. Morpheme generation rules give a surlace realization to molpho. logical tagis, such as P'ast, Ilon(orific), ctc. Rules for irregular verbs doal with final consonants and syllabification. Deletion rules eliminate one of two adjacent vowels on morpheme boundaries. Vowel harmony rules realize the harmonizing, archiphonemes $W U$ as o or wh and $E^{\prime}$ as $a$ or e repending on the quality of the preceding vowel. Contraction rules involve the merging of aljacent vowels to a diphthong or a single vowel as a result of the loss of the intervening syllable boundary.

Although it is possible in principle to write just one two-level rule system that describes all the alternations in parallel, it is very difficult in practice to create a rule systion with that degrec of complexity. 'The complexity arises from the fact that the formulation of every rute in a two-level system depends on overy rule that hits sone effect on the context of the rule that we are brying to express. l'or example, if there is a rulo that forees $X$ to be deleted in front of a $Y$ and another fule that introduces $\%$ between $X$ and $Y$, great care inust be exercised by the rule writer to make sure that, both rules are specified in a way that leaves room for the other rule to have its effect but does not depend on it if the deletion of $\mathrm{X}$ and the insertion of $\%$ are two independent alternations. 'The partioning of rules into sets and the interleaving of intersection and composition greatly simplifies the task of creating and updating the rules system. Rules that apply in different enviromments and do not affect each other can be compiled and intersected easily, whereas rules that involve alternations in overlapping contexts are most easily handled by placing them in diflerent levels in the cascade. In effect the rules are partially ordered. Sproat also noticed that rule interactions which may be casy to state in terms of ordered rules, are often much more diflicult to state in one two level rule system (Sproat, 1992).

for Korean, the partitioning of the rules for mor. phological altermations into the five sets described above appears to be the optimal choice. liach of the rules in the Hangul standard orthography published in March of 1988(Korcan Ministry of Lducation, 1988) is described in the corresponding two level rule sepa rately in our implernentalion. 'The order of rules takes the role of rule interactions. In this cascade, the alternations described in section 2 ass example (3) are split between three levels:

(8) (i) Rules for irregular predicates:

$\Lambda$ syllablo boundasy is introduced before the stem-linal $p$ in irregular - $p$ verbs/adjectives when a vowel-initial suflix follows. 'The following morpliene boumlary is deleted and $p$ is realized ats the hamonizing archiphoneme WU 
(ii) Vowel harmony rules:

(a) The WU is realized as $o$ if the vowel of the preceding surface syllable is [+light], otherwise $w u$

(b) 'The $E$ ' is realized as $a$ if the vowel of the preceding surface syllable is [+light], otherwise $e$.

(iii) Contraction rules:

'The morpherre boundary " + " can option ally bo deleted between $w u$ and $e$.

The effect of these rules with respect to the irregular $-p$ verb cwup 'to pick up' is shown in (9).

(9) (a) $c$ wu $\theta \quad p \quad\{p \mathrm{Verl}\}+E-s c$

(b) c wu-WU O OH-se

(c) cwu- wu 0 o $e-s e$

The intermediate level, (b), is eliminated in the cascade, thus the final lexical transducer maps (a) directly to (c).

\section{Conclusion}

The success of our work on Korean further underscores the point made by KKZ(Kartiunen, 1992a) that the most salient property of two-levol morphology is not the number of levels but the fact two-level rules describe regular relations (just like classical phonological rewrite rules) (Kaplan, 1988; Ritchie, 1992). Consequently, it is possible to combine sets of parallel two level rules by intersection and merge them with the lexicon and other rule systems in a cascade of compositions. 'The complexitics of Korean morphology make it desirable both for linguistic and computational reasons to allow for many more intermediate levels than assumed in previous works on Fnglish and French. Regardless of the number of intervening levels, the outcome is a single lexical transducer that directly maps lexical forms to their inflected surface realizations, and vice versa.

In the construction of the lexical automaton for $\mathrm{KO}$-. rean, we have put two-level rules to a novel use as wollformedness constraints on lexical forms. The sublexicons from which the $L A$ is constructed contain diacritic marks on the outer edge that identify the type of morphological constituents that the lexicon contains. The role of rules in the T.A constructions is to enforce morphotactics and, at the same time, to eliminate the diacritics that encode them.

'l'heoretically, we can get the same I'I' to compose the morphotactic and phonological rules all togetber into one rule and compose it with the initial $\mathbf{I} A$ or to compose the initial LA with each rule of the morphotactic and phonological rules one by one in order.
Practically, the composition of all the morphotactic and phonological rules into one rule causes the combinatorial explosion of states. This shows that ordered rules can be used to avoid the combinatorial explosion of states in one two level rule system too.

\section{References}

[1] Antworth, Evan I.(1990) PC-KIMMO: a twollevel processor for morphological analysis. Occasional Publications in Academic Computing, No. 16,Summer Institute of Iinguistics, Dallas, 'Iexas. 1990

[2] Kaplan, R. M.(1988) "Regular models of phonological rule systems". Alvey Workshop on Parsing and Pattern Recognition. Oxford University, $\Lambda$ pril, 1988

[3] Korean Ministry of Wducation.(1988) IIangul Standard Orhography (Revised in 1988), Documont number 88-1, Published in March 1988.

[4] Karttunen, Lauri, Kaplan, Ronald M., and Zaenen, Annie.(1992a) "Two-Jevel Morphology with Composition". Coling-92. Proceedings of the fifteenth International Conference on Computalional Iinguistics. Volume I. pp.141-148. 1992.

[5] Karltunen, Lauri and Bcesley, Kenneth R.(1992b) Two-Level Rule Compiler. Technical Report. Xerox Palo Alto Research Center. ISTI-92-2. October 1992. [P92-000149]. Palo Alto, California. 1992.

[6] Kartunen, Isauri.(1993) "linite-State Constraints". 'To appear in "The Last l'honological Rule, John Goldsmith, ed. Chicago University l'ress. Chicago. 1993.

[7] Kim, C.(1990) The Hxplanation of New Hangul Standard Orthography, Kul-Sup Press. Scoul. 1990.

[8] Koskenniemi, K.(1983) T'wo-level Morphology. A General Computational Model for Word-form Recognition and Production. Department of General Linguistics. University of IIelsinki. 1983.

[9] Kwon, II. and Chae, Y.(1991) "A DictionaryBased Morphological Analysis". Proceedings of the Natural Language Processing: Pacific Rim Symposium'91. pp.178-185, 1991.

[10] Ritchic, Gracme D.(1992) "languages Generated by Two-level Morphological Rules". Computational Linguistics, No. 18, Volume. 1, pp.41-59. March 1992.

[11] Sproat, R.(1992) Morphology and Computation, MIT press, 1992. 